REVUE HYBRIDE DE L'ÉDUCATION

\title{
Les dilemmes du métier des accompagnants des élèves à besoins éducatifs particuliers dans les écoles inclusives au Liban.
}

Basma Frangieh, T.I.E.S Education (Liban), Laboratoire ACTé (Université Clermont-Auvergne - France) basma.frangieh@ties-education.org Joumana Akiki, Université Libanaise-Faculté de Pédagogie jakiki@ul.edu.lb 


\section{REVUE HYBRIDE DE L'ÉDUCATION}

\section{Résumé}

La réalité sociale, culturelle et économique dans les écoles au Liban semble contribuer à la croissance des inégalités, qui devraient guider le nécessaire « changement de paradigme » que nécessite l'école inclusive. Notre intérêt porte sur l'étude du vécu des accompagnants scolaires des élèves à besoins éducatifs particuliers dans ces écoles inclusives.

L'objectif de cette recherche est de retenir les dilemmes du métier (Jorro, 2002) de ces professionnels. La méthodologie adoptée est celle de l'analyse de l'activité des pratiques (Clot, Faïta, Fernandez et Scheller, 2001). Nos résultats font apparaître quatre dilemmes du métier portant sur l'identité professionnelle de ces intervenants.

Mots-clés : accompagnant scolaire, besoins éducatifs particuliers, école inclusive, dilemme du métier, inégalité scolaire. 


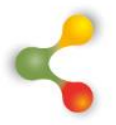

\section{REVUE HYBRIDE DE L'ÉDUCATION}

\section{Problématique}

Offrir une éducation de qualité pour tous les élèves dans les écoles au Liban constitue un défi majeur. En l'absence d'un cadre général et d'un plan d'action national, la mise en place d'une école inclusive y est jalonnée d'incertitudes, de tensions et de contradictions. L'éducation inclusive se propose de transformer l'école, " de la rendre accessible tant du point de vue des locaux que de l'enseignement ; elle affirme la nécessité d'un "changement de paradigme " avec l'ancienne logique "intégrative " (Bélanger, Frangieh, Graziani, Mérini et Thomazet, 2018). Elle exige un travail de fond pour tous les acteurs engagés. Cette perspective accentue l'importance du travail collectif qui constitue une pierre angulaire de l'école inclusive, rassemblant une multiplicité de partenaires ayant des expertises variées, appartenant à l'école et au secteur médico-social ${ }^{1}$. Elle entraîne la rencontre des acteurs et des professionnels qui appartiennent à deux mondes différents et qui ne sont pas habitués à œuvrer ensemble. Développer une école inclusive impliquerait donc la nécessité, d'abord, de développer une culture d'action conjointe ou, tout au moins, décider ensemble, tout comme cela implique de partager les responsabilités concernant tous les enfants (Cru, 1988). Cette dynamique traduit la croyance que l'éducation ne doit viser rien de moins que la pleine actualisation du potentiel de chaque élève (Landry, Ferrer et Vienneau, 2002). Cela exige, en premier lieu, un changement de culture de l'école et des institutions qui contribuent à la formation des personnes qui y œuvrent.

Parmi ces acteurs, retenons le recours aux accompagnants scolaires qui constitue une aide humaine assurant une présence au quotidien auprès des élèves ayant des besoins éducatifs particuliers (BEP) intégrés dans des classes ordinaires. Leur travail s'inscrit dans une démarche de collaboration avec les professionnels de l'école et du médicosocial. Toutefois, la présence de ces nouveaux " médiateurs 》 est actuellement la voie unique d'accès des enfants à besoins particuliers à l'école au Liban, une sorte de condition non avouée, indispensable à toute forme de scolarisation (Akiki et Frangieh, 2017).

L'accompagnement scolaire ne passe pas sans relever des difficultés et des défis majeurs à toutes les personnes entrant en jeu dans une école inclusive. Dans cette perspective, cette recherche s'intéresse à la situation de l'accompagnant scolaire dans cette nouvelle dynamique de travail, dans l'objectif de relever les dilemmes de leur métier dans la scolarisation des élèves ayant des BEP au sein d'écoles qui se considèrent inclusives.

\section{Cadre théorique}

\footnotetext{
${ }^{1}$ Ce sont des professionnels de la santé, de la rééducation, de l'éducation spécialisée et du travail social. 


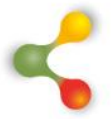

\section{REVUE HYBRIDE DE L’ÉDUCATION}

\section{L'accompagnement scolaire des élèves à besoins éducatifs particuliers}

Pour rendre accessible l'accueil des élèves ayant des BEP dans les établissements scolaires, le recours aux accompagnants scolaires a été une aide humaine apportée dans le cadre de la vie scolaire quotidienne. Les appellations utilisées pour désigner ces professionnels sont très diversifiées en fonction des pays, et leurs fonctions semblent varier entre l'aide humaine, l'assistance, le suivi, le soutien et l'accompagnement scolaire des élèves (Akiki et Frangieh, 2017). Quant au Liban, nous retenons également l'absence d'appellation unifiée pour désigner ces personnels, et souvent celles utilisées sont empruntées essentiellement aux langues française ou anglaise "shadow teacher", "AVS ", "learning support teacher", " enseignant de soutien », etc. (Akiki et Frangieh, 2017). Leurs fonctions peuvent dépasser le cadre de l'accompagnement, la communication et la socialisation, pour aborder le champ des apprentissages scolaires et les adaptations pédagogiques qui en découlent (Manansala et Dizon, 2008).

Toutefois, les limites entre leurs tâches et celles de l'enseignant de la classe sont controversées : l'AVS' (auxiliaire de vie scolaire) est un médiateur entre le maître et l'élève (Cochetel et Edouard, 2009), un accompagnant éducatif plutôt que pédagogique, même s'il participe parfois aux apprentissages (Philip, 2009). En fait, il assure une aide et un accompagnement en vue d'optimiser la scolarisation des enfants (Nédélec-Trohel et Toullec-Théry, 2010). Au sein de la classe, il est «le spécialiste du handicap » (ToullecThéry, 2012a, p.132), mais sa fonction ne se confond nullement avec celle d'un enseignant spécialisé (Belmont, Plaisance et Vérillon, 2011). L'AVS peut devenir un assistant d'enseignement, ce qui ne correspond pas à sa place dans la classe (Nédélec-Trohel, Joffredo-Lebrun et Magnen, 2012). II joue le rôle de médiation dans ses interactions orientées vers plusieurs acteurs : 'enseignant, l'enfant « en situation de handicap », mais aussi « les pairs d'âge ". Enseignant et AVS font preuve d'observation et d'adaptation pour guider leurs actions (Bedouin et Janner-Raimondi, 2016). Accompagner l'élève consiste à reformuler l'instruction dispensée par l'enseignant afin de la rendre compréhensible pour l'apprenant. II s'agit donc, pour l'accompagnant scolaire, de mettre à la portée de l'élève à besoins particuliers la situation d'apprentissage, dont la responsabilité incombe à l'enseignant.

Dans le pays de notre recherche, il semble difficile de parler de métier à proprement parler, reconnu, avec un profil bien établi, unifié pour tous les acteurs (enseignants, professionnels, parents, directeurs d'établissements, etc.), mais plutôt d'un " métier hybride ", sans identité réelle, en voie de construction en dehors de tout référentiel de compétences (Akiki et Frangieh, 2017). Les accompagnants scolaires sont à la recherche d'une identité professionnelle où les fonctions et les pratiques seraient

2 Dans cette recherche, l'appellation adoptée est celle d' " accompagnant scolaire " mais nous gardons également celles utilisées par les auteurs cités. 


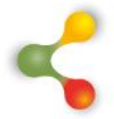

\section{REVUE HYBRIDE DE L'ÉDUCATION}

clairement définies. Le statut qui leur est réservé ne leur semble pas être à la hauteur de la tâche attendue, qui implique un fort engagement personnel et sollicite des compétences diverses. Être accompagnant scolaire demande " un fort investissement malgré un statut contesté » (Belmont, Plaisance et Vérillon, 2011, p.95).

L'enseignant quant à lui reste le seul responsable de la classe ; il joue le rôle de "technicien de la pédagogie » (Philip, 2009), mettant l'accompagnant dans une situation de dépendance à son égard. De ce fait, le travail d'accompagnement achevé par les AVS demanderait une « logique d'action commune » (Ebersold, 2009, p.19). L'AVS oscille entre quatre types de fonctionnement : une partition établie des rôles de chacun, une position surplombante de l'AVS, une position surplombante du professeur, ou une certaine symétrie entre les deux (Thoullec-Théry et Brissiaud, 2012). La précédente typologie est reconsidérée par Toullec-Théry (2012b) selon deux prototypes: des relations «égalitaires " entre les deux systèmes basées sur la coopération, et à l'inverse, des relations « inégalitaires » où la collaboration serait ponctuelle et source de conflits. Par conséquent, une insuffisante collaboration entre l'accompagnant, l'enseignant de la classe et l'école, influence négativement le succès des pratiques inclusives.

\section{Méthodologie : L'activité des accompagnants scolaires des élèves ayant des BEP dans les écoles au Liban}

\section{Sujets}

Cette recherche a été menée auprès de 89 accompagnants scolaires œuvrant dans des établissements scolaires qui se considèrent inclusifs et ceci dans trois régions du pays (Beyrouth, Liban-Nord et Liban-Sud). La répartition de l'échantillon est explicitée en détail dans la partie «procédure » présentée ci-dessous.

\section{Procédure}

Les données ${ }^{3}$ ont été recueillies entre avril 2016 et décembre 2017 et elles font l'objet de sept étapes :

1 ère étape : Étape préliminaire

II s'agissait d'entretiens individuels effectués auprès de cinq accompagnants scolaires d'élèves ayant des BEP dans l'objectif de cerner la réalité du vécu de ces professionnels dans les écoles. Cette étape a permis de compléter les informations recueillies et d'avoir des données en lien avec le contexte libanais.

2ème étape : Élaboration du questionnaire

En nous basant, d'une part, sur les données théoriques en lien avec

3 Les trois premières étapes de notre recherche ont constitué l'objet d'une autre publication : "Accompagnants scolaires et école inclusive. Cas du Liban » (Akiki et Frangieh, 2017).

Volume 3; Numéro 1 $43 \quad$ Printemps 2019




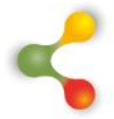

\section{REVUE HYBRIDE DE L'ÉDUCATION}

la profession des accompagnants scolaires et d'autre part, sur l'analyse des cinq entretiens, un questionnaire a été élaboré. Avant de procéder aux passations du questionnaire, nous avons eu recours à un pré-test effectué auprès d'une dizaine d'accompagnants scolaires, afin de vérifier la formulation des questions et d'y apporter, le cas échéant, les modifications souhaitables.

\section{3ème étape : Passation du questionnaire}

Nous avons procédé à la distribution des questionnaires aux accompagnants scolaires dans les écoles ayant accepté de participer à la recherche. L'objectif était de recueillir des données en lien avec la nature de l'activité et des fonctions exercées, de même que les difficultés et les besoins rencontrés.

À la suite du dépouillement des 78 questionnaires retenus, il s'est avéré que les résultats liés à nos points d'intérêt ne reflétaient pas la pratique au quotidien des accompagnants scolaires. Nous avons alors décidé d'approfondir cette recherche en ayant recours à la méthodologie de l'analyse de l'activité (Clot, 2001) de ces professionnels, où l'image est utilisée comme support principal des observations.

4ème étape : Filmer des séances classes/ tournage en salle de classe

Trois séances classes ont été filmées dans deux établissements scolaires auprès de trois accompagnants, deux parmi eux assurant le suivi d'un seul élève intégré dans une classe régulière, et le dernier œuvrant auprès d'un groupe d'élèves ayant des BEP accueilli dans une classe avec les élèves tout venant.

5ème étape : Réalisation des auto-confrontations simples

Après chaque tournage de séance classe, un entretien d'autoconfrontation simple a été réalisé auprès des accompagnants scolaires filmés. Cette étape consiste à les faire visionner un film de leur propre pratique professionnelle, afin qu'ils puissent commenter et expliquer leurs actions. II ne s'agit pas, à ce stade, de construire la professionnalité, mais de comprendre ce qui s'oppose à son élaboration; d'entamer une revue en confiance de l'activité menée afin de provoquer un processus réflexif permettant de dégager des pratiques professionnelles souhaitables.

\section{6ème étape : Réalisation des allo-confrontations}

Huit allo-confrontations ont été mises en place. Cette étape consiste à utiliser les trois séances filmées comme déclencheurs d'entretiens sur l'activité des accompagnants scolaires non filmés afin qu'ils puissent s'exprimer au sujet de leur métier en se basant sur ce que font les autres collègues et en comparant l'activité des autres à leurs propres pratiques.

\section{7 ème étape : Réalisation des auto-confrontations croisées}

Trois auto-confrontations croisées ont été achevées auprès de huit accompagnants scolaires. Elles consistent à confronter les pairs de même niveau d'expertise aux activités de leurs collègues filmés, dans l'objectif de 


\section{$\&$}

\section{REVUE HYBRIDE DE L'ÉDUCATION}

partager ensemble les observations relevées lors du visionnage des moments considérés comme essentiels par les acteurs observés. Les questionnements émergent du plan personnel pour se préciser en dispositions professionnelles et s'orienter vers la mise en œuvre de situations d'apprentissage, ce qui favorise le développement des échanges (Amigues, Faïta et Saujat, 2004).

\section{Analyse des données}

En référence à Bardin (1991), trois phases ont été effectuées pour traiter les données de cette recherche :

- La thématisation : Suite aux auto-confrontations simples, aux alloconfrontations et aux auto-confrontations croisées, nous avons procédé au décodage des réponses données par les accompagnants scolaires des élèves ayant des BEP. Un tableau a été élaboré contenant tous les verbatim en lien avec leur travail (activité).

- L'organisation : Ces répliques sont rangées et regroupées sous des thématiques.

- La sélection: Pour avancer dans notre réflexion, quatre thématiques ont été choisies en lien avec les difficultés recueillies par les accompagnants scolaires des élèves ayant des BEP au regard de leurs activités (métier) en contexte d'école inclusive, et nous les avons formalisées en dilemmes.

\section{Résultats}

Suite à la méthodologie appliquée, quatre dilemmes du métier de l'accompagnant scolaire ont été décelés dans ses interventions dans les écoles inclusives au Liban. Les résultats sont présentés ci-après sous la forme d'une analyse, étayée par des extraits de verbatim ${ }^{4}$.

\section{Suivi d'un seul élève ou plusieurs}

En dehors de tout cadre législatif organisant le travail des accompagnants scolaires dans notre pays, la diversité des pratiques sur le terrain semble être de règle, de même que la variété des "formules " adoptées au sein des écoles. La majorité des accompagnants interviewés interviennent auprès d'un seul élève "je travaille en individuel »; ils considèrent que la nature de leur activité l'exige "l'accompagnant est la personne spécialisée dans le suivi d'un seul élève en classe ».

\footnotetext{
${ }^{4}$ Les parties entre guillemets et en italique sont extraites des verbatim de nos enregistrements effectués auprès des accompagnants scolaires qui ont accepté de participer à la réalisation de cette recherche ; elles sont traduites de l'arabe.
} 


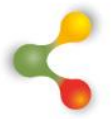

\section{REVUE HYBRIDE DE L’ÉDUCATION}

Or, parmi les accompagnants scolaires des élèves ayant des BEP rencontrés, certains $(n=4)$ assurent le suivi de plusieurs élèves à la fois au sein de la même classe. Cette situation semble difficile pour eux, voire impossible à gérer : "Impossible que l'accompagnant puisse s'occuper de plus d'un seul élève... ", " un seul élève exige déjà beaucoup de temps... entre le suivi de l'élève en classe et les séances d'activités à assurer en dehors de la classe... ", "à la limite, ce serait possible pour deux élèves, pas plus, et à condition qu'ils aient la même difficulté, et qu'ils fassent partie de la même classe". La difficulté réside dans les rythmes différents d'apprentissage, le type de l'intervention (en classe ou hors classe), mais aussi au niveau des besoins qui ne sont pas identiques pour tous les élèves. De plus, œuvrer auprès de plusieurs élèves en même temps mène à un questionnement au niveau de l'organisation de leur travail : intégrer les élèves au sein du groupe classe ou travailler séparément avec eux " au début de l'année, je travaillais avec cinq élèves [...]. C'était difficile à gérer, en plus on était mis à l'écart, il y avait la classe et nous. " La proposition des activités trop détachées de celles du groupe classe conduit l'élève à une situation d'isolement et nuit à son rendement scolaire.

Ces professionnels sont-ils en devoir d'intervenir auprès d'un seul élève ou auprès d'un groupe ? Cette question, soulevée par les éléments présentés ci-dessus, révèle un véritable dilemme du métier des accompagnants dans leurs interventions dans les écoles inclusives : "suivi d'un seul élève » ou « suivi de plusieurs ».

\section{Profession de l'enseignant ou profession en voie de construction}

Le métier que j'exerce diffère de celui de l'enseignant de la classe [...] Je ne crois pas qu'il devrait y avoir un métier d'accompagnant scolaire des élèves ayant des $B E P$. Son travail est identique à celui de tout enseignant à l'école.

Entre ces deux extrêmes, où se situent les pratiques ? Le premier point de vue consiste à avancer que les deux professions sont distinctes : " mon rôle ne se confond pas avec celui de l'enseignant ; j'ai mon travail, elle a le sien ». En dehors de l'élève ayant des BEP, " chacune a son travail et je n'interfère pas au niveau du sien. "Le rôle de l'accompagnant est d'assurer un soutien à l'enseignant. Ce dernier "ne peut pas s'occuper de la classe et de l'enfant ayant des BEP en même temps. " II est le “ médiateur » entre, d'une part, l'élève, et d'autre part, l'enseignant et les autres élèves de la classe. Son champ d'intervention est complètement différent de celui de l'enseignant : "en plus de l'aspect académique, je lui communique les bases de la vie sociale ", " aucun travail n'est exigé de ma part auprès des autres élèves de la classe ».

Les fonctions s'effectuent souvent en dehors d'un profil de poste bien établi, "jusqu'à présent, il n'existe pas de description claire des compétences requises pour l'exercice de cette profession. " La formation

Volume 3; Numéro $1 \quad 46 \quad$ Printemps 2019




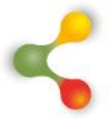

\section{REVUE HYBRIDE DE L'ÉDUCATION}

initiale ne semble pas non plus constituer une condition essentielle pour occuper cette fonction. "On m'a embauchée parce qu'ils ne réussissaient pas à trouver quelqu'un, alors que ma formation de base n'a rien à voir avec le domaine de l'éducation. " L'expérience se chargera du reste, "avec le temps, on apprendra ce qu'il faut faire, comme n'importe quel autre métier simple à exercer. »

Quant à la répartition des tâches, les accompagnants estiment que "l'enseignante de la classe est la personne principale. " Une sorte de dépendance est perçue dans cette relation en binôme, "l'enseignante est le «patron" et à vous de savoir comment traiter avec elle." Les accompagnants estiment que leur métier n'est pas reconnu : "On ne nous accorde pas du tout de l'importance ", «il existe cette perception que l'accompagnateur ne fait rien ", "je ne me suis pas sentie appréciée, en toute franchise. C'est un métier où l'on ne progresse pas ».

L'autre point de vue consiste à considérer que l'accompagnant est un enseignant comme les autres. Aucune différence n'est retenue dans l'exercice des fonctions, "je suis une enseignante ordinaire ; mes élèves sont plus faibles que les autres, ont moins de capacités, c'est tout ", "je suis encore une simple enseignante ", "ma relation avec l'enseignante est une relation d'égal à égal ", "les responsables nous traitent comme des enseignants ordinaires ". D'ailleurs, une minorité $(n=3)$ a noté que le contrat avec l'école est un contrat d'enseignant. Mais cette perception de l'équité entre accompagnant et enseignant est loin de constituer l'unanimité auprès des personnes concernées.

En conséquence, les données exposées ci-dessus ont donc fait surgir un deuxième dilemme du métier des accompagnants scolaires : estce une «profession de l'enseignant» ou une «profession en voie de construction »?

\section{Travail en équipe ou travail en individuel}

La plupart des accompagnants scolaires interrogés sont laissés à leur sort; ils œuvrent seuls dans leur coin, "chacun travaille de son côté ", "je travaille seule à l'école", "je n'ai pas de relation avec les professionnels de l'école, je bosse seule avec l'élève ", " absence de travail d'équipe ». Vu le manque de collaboration et d'organisation, "pas d'organisation ", " absence de travail d'équipe », entre les accompagnants et les professionnels de l'école (enseignants et équipe pluridisciplinaire), les interviewés ont exprimé leurs demandes d'organiser leur travail, "il faut qu'il $y$ ait une organisation/coordination ", " personne n'a défini mon travail, j'ai élaboré moi-même les objectifs à travailler ", "il faudrait que le shadow teacher coordonne son travail avec l'équipe pluridisciplinaire ». Les parents constituent pour ces accompagnants une pierre angulaire dans l'éducation de leurs enfants, "il faut qu'il y ait une collaboration avec les parents ». Les accompagnants se sentent délaissés, seuls avec l'élève ayant des BEP , " je n'ai aucune relation avec l'école, je suis seule avec l'élève », ils sont 


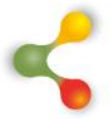

\section{REVUE HYBRIDE DE L'ÉDUCATION}

devenus débrouillards, " tout le temps, je suis seule, je me débrouille toute seule ».

D'autres accompagnants manifestent une collaboration continue avec les enseignants de la classe, surtout quand ils interviennent auprès de l'élève hors de la classe " je suis en relation continue avec l'enseignant pour voir ce qu'ils ont appris de nouveau en notre absence et le tenir au courant des acquis et non acquis des élèves ". Les échanges non formels avec les enseignants et l'équipe pluridisciplinaire sont primordiaux pour aborder le cas de certains élèves, " on organise toutes les semaines, durant le break, une réunion pour discuter de certains cas ». Les réunions formelles sont également exigées, "tous les vendredis on se réunit pour organiser le travail, on rédige un rapport général sur l'ensemble des tâches effectuées pour l'adresser aux parents ". Ces deux temps d'échange permettent à chaque professionnel de donner des pistes d'intervention en lien avec son champ d'expertise.

L'adaptation des notions se fait parfois en collaboration avec l'enseignant de la classe, "l'enseignante me remet les explications de la leçon, et moi je les répète pour mon élève avec ma méthode personnelle ". Cette réflexion soulève donc un troisième dilemme du métier des accompagnants scolaires : " travail en équipe » ou « travail en individuel », doivent-ils travailler en binôme avec l'enseignant, ou en tant que professionnels dont les tâches et responsabilités sont clairement différentes de celles de l'enseignant? Les avis des accompagnants scolaires sont partagés, surtout s'ils considèrent que leur métier est une profession en soi, bien distincte de celle de l'enseignant.

\section{Prise de décision personnelle ou conformité à une décision de l'établissement scolaire}

La majorité des accompagnants scolaires interrogés se sentent marginalisés et mis à l'écart dans la classe, "moi et l'élève sommes tous deux dans la classe, mais mis à l'écart ", " c'est comme si nous étions dans une autre classe ... Le shadow teacher est l'enseignant de l'élève ayant des $B E P$ et les autres élèves ont un autre enseignant qui est celui de la classe ». Cette situation les encourage à prendre leurs décisions seuls, sans avoir recours à l'enseignante de la classe, "ils m'ont rien dit quoi travailler », " ils m'ont fait comprendre que " ça se passe entre toi et l'élève, c'est à toi de te débrouiller » ", "sans recourir souvent à l'enseignant ». Ils ont l'impression d'être parachutés sur le terrain, sans consignes claires.

Ils leur incombent aussi d'organiser la journée de l'élève ayant des BEP. Dans quel endroit intervenir auprès de l'élève, est-ce en classe ou hors de la classe? "Si je veux travailler avec lui en dehors de la classe, personne n'intervient ", " je prends seule la décision de la/le faire sortir de la classe ", " personne n'intervient si je travaille avec l'élève dans la classe ou hors de la classe ». 


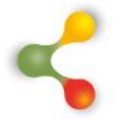

\section{REVUE HYBRIDE DE L'ÉDUCATION}

Dans la majorité des cas, la présence des accompagnants scolaires au sein de l'établissement scolaire et dans l'équipe pluridisciplinaire n'est pas très valorisée, leurs points de vue et leurs avis ne sont pas pris en considération, "on n'écoute pas votre point de vue au niveau de l'équipe de travail, on n'en tient pas compte ". De même, ils ne sont pas invités à participer aux réunions d'équipe organisées par l'établissement scolaire, ils ne sont également pas concernés par les décisions prises en lien avec l'élève suivi. De façon générale, les contacts avec les professionnels de l'école et du médico-social semblent être inexistants ou encore jugés superficiels.

Tandis que d'autres accompagnants scolaires, qui constituent une minorité, trouvent un meilleur soutien de la part de leur direction et de leur responsable. Les décisions sont prises ensemble, mais toujours sans la contribution et l'intervention de l'enseignant de la classe, "les décisions se prennent en accord avec le responsable ou le directeur. L'enseignant n'intervient pas ».

Ces professionnels sont-ils censés avoir recours à un tiers pour prendre les décisions ? Faut-il qu'il y ait un règlement conforme pour tous les professionnels de l'école y compris les accompagnants scolaires?

Ces données font apparaître un quatrième dilemme du métier de l'accompagnant scolaire, à savoir "prise de décision personnelle » ou " conformité à une décision de l'établissement scolaire ».

\section{Discussion}

\section{Premier dilemme}

Aborder la thématique de l'inclusion scolaire suppose mettre au premier plan de la réflexion l'élève concerné lui-même, " acteur du système d'échanges ", et ayant "le statut de go-between, message et messager entre sa famille et l'école et, par extension, les autres espaces d'accompagnements et de soins » (Perrenoud, 1987, cité par Champagnac et Mérini, 2017, p.157). L'accompagnant scolaire trouve sa place au sein de ces différents espaces, et semble devenir une condition sine qua non de la scolarisation en milieu ordinaire des élèves ayant des BEP. II peut intervenir auprès d'un seul ou de plusieurs élèves d'une même classe. Toutefois, le modèle le plus observé dans les écoles libanaises est le suivi en individuel. Situation à double tranchant dans la mesure où ce binôme élèveaccompagnant constitue un "nous " face à l'entité représentée par l'enseignant et les autres élèves de la classe. L'accompagnant scolaire assurant le suivi de plusieurs élèves se trouve dans l'obligation de gérer des situations différentes (tels que des rythmes d'apprentissage différents, présence dans/hors de la classe), nécessitant le déploiement d'efforts supplémentaires et un travail d'adaptation continuel. Ce qui crée parfois « un sentiment de solitude, dans un travail pourtant éminemment relationnel » (Allenbach, 2015, p.25). 


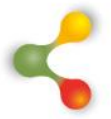

\section{REVUE HYBRIDE DE L'ÉDUCATION}

\section{Deuxième dilemme}

L'accompagnant scolaire remplit des rôles et des fonctions qui ne sont pas toujours établis clairement et exerce parfois sa fonction en dehors de toute formation ou expériences antérieures. Rares sont les écoles qui remettent aux accompagnants scolaires un profil de poste déterminant les compétences requises, leurs tâches et responsabilités auprès de l'élève. L'accompagnant oscille entre deux pôles : serait-il un enseignant à part entière, ou bien un assistant à l'enseignant jouant le rôle de soutien ou de médiateur auprès de l'enfant? En fait, le champ de ses compétences sont variées: pédagogiques, didactiques, relationnelles, en plus des connaissances relatives aux caractéristiques des élèves, et une vision de la diversité encourageant le travail en partenariat (Grangé et Dubé, 2015). Dans la pratique, les champs d'intervention sont distincts, voire « inégalitaires », mais nécessitent un travail de concertation continuel.

La reconnaissance de cette fonction et sa construction comme métier spécifique s'imposent: " un métier n'est pas seulement une «pratique » » ou une activité (Clot, 2007, p.86). De ce fait, c'est bien une fonction nouvelle qui apparaît attribuée aux AVS (non issus du corps enseignant), renforçant le phénomène de divisions du travail éducatif, mais nécessitant aussi des cohésions d'actions entre les différents intervenants. Ces derniers ne devraient plus se percevoir comme "des personnels seconds » (Belmont, Plaisance et Vérillon, 2011, p.101), ni se poser des questions quant à la légitimité et l'utilité de leur présence auprès de l'enfant. Nous assistons de nos jours à un développement de nouvelles professionnalités dans les classes. II s'impose donc de « repenser le travail enseignant dans sa compétence de travail en partenariat », et en particulier avec celui des accompagnants scolaires (Barthélémy, 2014, p.240).

\section{Troisième dilemme}

L'un des éléments incontournables pour la mise en œuvre de l'école inclusive est le partenariat. Dans cette perspective l'engagement individuel des enseignants en lui seul n'est pas suffisant. Pour favoriser la scolarisation des élèves ayant des BEP, l'accompagnant scolaire constitue une aide et une ressource humaine (Guirimand et Mazereau, 2016) non négligeable et précieuse pour la réussite de l'élève. II va devoir travailler avec l'enseignant dans la classe. II est donc préférable et pour eux, et pour l'enfant, que la relation se passe favorablement.

Ainsi, l'enseignant doit prévoir, en collaboration avec l'accompagnant scolaire et en fonction de chaque situation d'apprentissage, les interventions particulières auprès des élèves ayant des BEP. Si cette assistance est proposée, c'est bien parce que les enseignants dits " ordinaires " ne sont pas en mesure de jouer ce rôle attendu. C'est, en effet, en concertation avec l'enseignant et sous sa responsabilité pédagogique que l'accompagnant scolaire devra œuvrer. II est sous la 


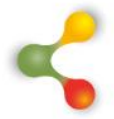

\section{REVUE HYBRIDE DE L’ÉDUCATION}

responsabilité pédagogique de l'enseignant. Cela ne veut pas dire que l'accompagnant doit se mettre lui-même en retrait de l'enseignant et des autres élèves, comme cela se produit parfois dans certaines classes. Les situations d'apprentissage doivent être anticipées conjointement par les binômes accompagnant scolaire et enseignant. Cette collaboration est une sorte de " carburant " nécessaire à une école plus inclusive (Allenbach, Duchesne, Grémion et Leblanc, 2016, p.105). L'enseignant, de son côté, n'opère plus en " soliste "; il se transforme en un " chef d'orchestre, garant du système didactique » (Cochetel, 2017, p.55). C'est bien une collaboration qu'il convient de promouvoir «au lieu d'un clivage entre les deux professionnels » (Philip, 2009, p.43), ainsi qu'une convergence des intérêts et des attitudes.

\section{Quatrième dilemme}

L'accompagnant scolaire ne trouve pas toujours de réponse pour pallier à toutes les difficultés du quotidien.

La présence d'un autre adulte dans la classe ne doit pas conduire l'élève dans une situation d'isolement de par des mises en activités pédagogiques trop détachées de celles du groupe classe ou du fait d'une posture de l'accompagnant faisant obstacle à la mission pédagogique de l'enseignement.

Par conséquent, il serait primordial d'éviter que les élèves " intégrés » ne reçoivent bien souvent, tel que le soulève Armstrong (2000), le statut de "visiteurs » plutôt que de membres à part entière de la communauté scolaire. L'école semble ainsi renforcer les discriminations et, par conséquent, les inégalités entre élèves au sein des classes à travers ses pratiques éducatives, entre autres. Les élèves accompagnés d'un adulte sont traités différemment en matière d'enseignement. Or, peut-on lutter contre les inégalités de scolarisation au travers d'un traitement préférentiel et d'une action " ciblée " consistant à donner " quelque chose de plus (ou de « mieux » ou de « différent ») » (Frandji et Rochex, 2011, p.101) à ces élèves ayant des BEP?

En effet, le développement des relations avec leurs partenaires se révèle primordial pour permettre à ces professionnels de comprendre et maîtriser leur fonction d'accompagnement. C'est grâce à ces échanges qu'ils parviennent à trouver leur place par rapport aux autres acteurs et à déterminer progressivement leur champ d'action. En cela, l'expérience de ces acteurs renvoie à ce que Ebersold (2003) nomme un "système équitable de coopération ».

\section{Conclusion}

Les accompagnants scolaires constituent une pierre angulaire en termes de réponse humaine malgré leur situation de professionnalisation 


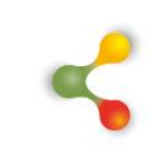

\section{REVUE HYBRIDE DE L'ÉDUCATION}

inachevée. Ils s'investissent au profit de l'élève accompagné, afin de répondre à ses besoins de compensation dans un contexte inclusif.

Notre recherche nous a permis, d'une part, de relever quelques dilemmes du métier des accompagnants scolaires qui entravent l'exercice de leurs activités et, d'autre part, de traiter un ensemble de questions relatives à certains aspects de l'exercice qui semblent être très compliqués à gérer, voire parfois irréalisables. Malgré les progrès remarqués ces dernières années, notre recherche montre qu'il reste beaucoup de chemin à parcourir dans cette voie : il s'agit de passer d'une activité mal reconnue, mal rémunérée, accomplie dans un temps limité, au statut incertain, à une activité qui serait socialement organisée et reconnue dans un espace social de référence, qui reposerait sur « un système de règles octroyant un statut à ceux qui exercent l'activité et indiquant des conditions d'accès, notamment en termes de formation " (Cadet, 2008, p.340). Pour ce faire, certaines actions semblent être nécessaires. Notons à titre d'exemple l'élaboration d'une fiche de poste pour les accompagnants scolaires dans l'objectif d'organiser leur travail, la professionnalisation de leur activité afin de trouver une visibilité, une identité professionnelle, et la mise en place de formations favorisant la reconnaissance du métier dans le but d'obtenir un diplôme reconnu. 


\section{REVUE HYBRIDE DE L'ÉDUCATION}

\section{Références}

Akiki, J. et Frangieh, B. (2017). Accompagnants scolaires et école inclusive. Cas du Liban. Beau Bassin, Mauritius: Éditions Universitaires Européennes.

Allenbach, M. (2015). Intervenir à l'école : les défis de la collaboration. Revue suisse de pédagogie spécialisée, 1, 21-28.

Allenbach, M., Duchesne, H., Grémion, L. et Leblanc, M. (2016). Le défi de la collaboration entre enseignants et autres intervenants dans l'école inclusive : croisement des regards. Revue des sciences de l'éducation, 42(1), 86-121.

Amigues, R., Faïta, D. et Saujat, F. (2004). « L'autoconfrontation croisée » : une méthode pour analyser l'activité enseignante et susciter le développement de l'expérience professionnelle. Bulletin de psychologie, 469, 41-44.

Armstrong, F. (2000). "Les paradoxes de l'éducation inclusive en Angleterre ». Dans M. Chauvière et É. Plaisance (dir.). L'école face aux handicaps. Éducation spéciale ou éducation intégrative? (pp. 118-132). Paris, France : Presses universitaires de France.

Bardin, L. (1991). L'analyse de contenu. Paris, France: Presses universitaires de France.

Barthélémy, V. (2014). Professeur des écoles dans une école inclusive : vers une nouvelle professionnalisation dans la construction bipartite enseignant/AVS. La nouvelle revue de l'adaptation et de la scolarisation, 4(68), 239-252.

Bedouin, D. et Janner-Raimondi, M. (2016). Rôles de l'auxiliaire de vie scolaire auprès de jeunes enfants en situation de handicap. Spirale. Revue de recherches en éducation, 57, 67-80.

Bélanger, J., Frangieh, B., Graziani, E., Mérini, C. et Thomazet, S. (2018). L'école inclusive : derrière les mots quelles conceptions et quel recours aux différentes formes de «l'agir ensemble ». Revue des sciences de l'éducation, 44(1), 138-165

Belmont, B., Plaisance, E. et Vérillon, A. (2011). Conditions d'emploi des auxiliaires de vie scolaire et qualité de l'accompagnement des élèves handicapés. Revue française de pédagogie, 174, 91-106.

Cadet, J.-P. (2008). «Qu'est-ce que la professionnalisation? » Dans J.J. Paul et J. Rose (dir.). Les relations formation-emploi en 55 questions (pp. 339-344). Paris, France : Dunod. 


\section{REVUE HYBRIDE DE L'ÉDUCATION}

Champagnac, P. et Mérini, C. (2017). La coopération liée aux situations de handicap. Partenariats et non-dits, La nouvelle revue de l'adaptation et de la scolarisation, 1(77), 153-168.

Clot, Y. (2001). Psychopathologie du travail et clinique de l'activité. Éducation permanente. Clinique de l'activité et pouvoir d'agir, 146, 35-50.

Clot, Y. (2007). De l'analyse des pratiques au développement des métiers. Éducation et didactique, 1(1). Repéré à http://educationdidactique.revues.org/106

Clot, Y., Faïta, D., Fernandez, M. et Scheller, L. (2000). Les entretiens en autoconfrontation croisée : une méthode en clinique de l'activité. Pistes, revue électronique, 1 , mai. Repéré à http://www.pistes.uqam.ca/v2n1/sommaire.html

Cochetel, G. (2017). AESH et enseignant. Collaborer dans une école inclusive. Paris, France : Canopé.

Cochetel, G. et Edouard, M. (2009). Auxiliaire de vie scolaire : accompagner l'élève handicapé. Clermont-Ferrand, France : CRDP d'Auvergne.

Cru, D. (1988). Collectif et travail de métier. Dans C. Dejours (Éd.). Plaisir et souffrance dans le travail (pp. 43-49). Paris, France : Editions de l'AOCIP.

Ebersold, S. (2003). L'intégration scolaire, un système équitable de coopération à construire. European Journal of Special Needs Education, 18(1), 89-107.

Ebersold, S. (2009). Autour du mot inclusion, Recherche et Formation, 2(61), 71-83.

Frandji, D. et Rochex, J.Y. (2011). De la lutte contre les inégalités à l'adaptation aux « besoins spécifiques ». Éducation et formations, 80, 95-108.

Grangé, N. et Dubé, F. (2015). Définir la fonction d'enseignant-ressource dans le secondaire : une recherche-action formation. La nouvelle revue de l'adaptation et de la scolarisation, 2(70-71), 121-136.

Guirimand, N. et Mazereau, P. (2016). Inclusion scolaire et professionnalités enseignantes entre attentes et contradictions. Carrefours de l'éducation, 2(42), 47-60.

Jorro, A. (2002). Professionnaliser le métier d'enseignant. Revue Européenne de Psychologie Appliquée, 54(2), 101-108. 


\section{REVUE HYBRIDE DE L'ÉDUCATION}

Landry, R., Ferrer, C. et Vienneau, R. (2002), La pédagogie actualisante. Éducation et francophonie, 30(2), 255-284.

Manansala, M.E. et Dizon, E.I. (2008). Shadow teaching scheme for children with autism and attention deficit-hyperactivity disorder in regular schools. Education quarterly, 66(1), 34-49.

Nédélec-Trohel, I., Joffredo-Lebrun, S. et Magnen, M. (2012). Étude de pratiques inclusives en classe ordinaire : rôle et place de l'AVS vis-à-vis du professeur. La nouvelle revue de l'adaptation et de la scolarisation, 1(57), 155-166.

Philip, C. (2009). Auxiliaire de vie scolaire, une fonction paradoxale ? La nouvelle revue de l'adaptation et de la scolarisation, 1(45), 33-44.

Toullec-Théry, M. (2012a). Scolarisation de trois élèves autistes à l'école primaire : positionnements des enseignants et des auxiliaires de vie scolaire. La nouvelle revue de l'adaptation et de la scolarisation, 4(60), 129-142.

Toullec-Théry, M. (2012b). Les relations entre enseignant et Auxiliaire de Vie Scolaire dans la scolarisation des élèves en situation de handicap, [en ligne], Notes du CREN (Centre de Recherche en Éducation de Nantes), 10, Avril.

Toullec-Théry, M. et Nédélec-Trohel, I. (2010). École et inclusion. Recherche et formation, 64, 123-138.

Toullec-Théry, M. et Brissiaud, M. (2012). Scolarisation d'un élève en situation de handicap: le cas d'un accompagnement délicat effectué par un Auxiliaire de vie scolaire (AVS). La nouvelle revue de l'adaptation et de la scolarisation, 1(57), 139-153. 